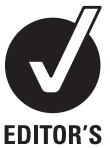

CHOIC

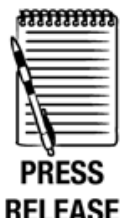

RELEASE
- Additional figures are published online only. To view these files please visit the journal online (http://heart.bmj. com)

${ }^{1}$ Coronary Care Unit, Cardiovascular Institute, Hospital Clínico San Carlos, Madrid, Spain

${ }^{2}$ Centro Nacional de Investigaciones Cardiovasculares Carlos III (CNIC), Madrid, Spain

\section{Correspondence to} Borja Ibanez, Imaging in Experimental Cardiology Laboratory, Atherothrombosis and Imaging Department, Centro Nacional de Investigaciones Cardiovasculares Carlos III (CNIC), c/Melchor Fernández Almagro, 3, Madrid 28029, Spain; bibanez@cnic.es

Accepted 1 March 2011 Published Online First 27 April 2011

\title{
Circadian variations of infarct size in acute myocardial infarction
}

\author{
Aida Suárez-Barrientos, ${ }^{1}$ Pedro López-Romero, ${ }^{2}$ David Vivas, ${ }^{1,2}$ \\ Francisco Castro-Ferreira, ${ }^{1}$ Ivan Núnez-Gil, ${ }^{1,2}$ Eduardo Franco, ${ }^{1}$ Borja Ruiz-Mateos, ${ }^{1}$ \\ Juan Carlos García-Rubira, ${ }^{1}$ Antonio Fernández-Ortiz, ${ }^{1}$ Carlos Macaya, ${ }^{1}$ Borja Ibanez ${ }^{1,2}$
}

\begin{abstract}
Background The circadian clock influences a number of cardiovascular (patho)physiological processes including the incidence of acute myocardial infarction. A circadian variation in infarct size has recently been shown in rodents, but there is no clinical evidence of this finding.

Objective To determine the impact of time-of-day onset of ST segment elevation myocardial infarction (STEMI) on infarct size.
\end{abstract}

Methods A retrospective single-centre analysis of 811 patients with STEMI admitted between 2003 and 2009 was performed. Infarct size was estimated by peak enzyme release. The relationship between peak enzyme concentrations and time-of-day were characterised using multivariate regression splines. Time of STEMI onset was divided into four 6-hour periods in phase with circadian rhythms.

Results Model comparisons based on likelihood ratio tests showed a circadian variation in infarct size across time-of-day as evaluated by peak creatine kinase (CK) and troponin-I (Tnl) concentrations $(p=0.015$ and $p=0.012$, respectively). CK and Tnl curves described similar patterns across time, with a global maximum in the 6:00-noon period and a local minimum in the noon-18:00 period. Infarct size was largest in patients with STEMI onset in the dark-to-light transition period (6:00-noon), with an increase in peak CK and Tnl concentrations of $18.3 \%(p=0.031)$ and $24.6 \%$ $(p=0.033)$, respectively, compared with onset of STEMI in the 18:00-midnight period. Patients with anterior wall STEMI also had significantly larger infarcts than those with STEMI in other locations.

Conclusions Significant circadian oscillations in infarct size were found in patients according to time-of-day of STEMI onset. The infarct size was found to be significantly larger with STEMI onset in the dark-to-light transition period (6:00-noon). If confirmed, these results may have a significant impact on the interpretation of clinical trials of cardioprotective strategies in STEMI.

\section{INTRODUCTION}

It is well established that the circadian rhythm influences cardiovascular system physiology, inducing diurnal variations of blood pressure, heart rate, cardiac output and endothelial function among other physiological parameters. ${ }^{1}$ It is also known that the incidence of different pathological cardiovascular events (such as myocardial infarction (MI), sudden cardiac death, arrhythmias) exhibits a marked time-of-day dependence in humans, with higher incidences around the sleep-to-wake transition period..$^{2}$ Although the intrinsic mechanisms of these phenomena remain uncertain, it has been speculated that the association between cardiovascular events and circadian variation could be ascribed to extracardiac factors such as oscillations in epinephrine and cortisol levels, prothrombotic factors, platelet aggregation and coronary arterial flow, among others. ${ }^{3}$ More recently it has been shown that a molecular mechanism intrinsic to the cardiomyocyte such as the circadian clock might contribute to cardiovascular disease. ${ }^{4}$

Epidemiological studies have consistently shown that the onset of an acute myocardial infarction (AMI) significantly varies throughout the day, with an obvious peak incidence in the dark-to-light transition period (ie, early morning hours). ${ }^{5} 6$ Conversely, the effect of the time of AMI onset on the final infarct size has not been systematically explored. In mammalian hearts during an AMI there is activation of an intrinsic defence mechanism encompassed by several protein pathways. ${ }^{7}$ The survival of ischaemic myocytes (cardioprotection) depends to some extent on the degree of activation of these salvage pathways. Preclinical studies have shown that the expression of some proteins from these survival pathways has a robust circadian oscillation. ${ }^{9}$ It is therefore plausible that circadian fluctuations of such myocardial salvage mechanisms might result in variation in the tolerance of the heart to ischaemia/reperfusion (I/R).

It has recently been shown for the first time that there is an association between time-of-day and intrinsic cardioprotection in mice subjected to experimental AMI. ${ }^{10}$ This variation in the spontaneous tolerance to I/R was regulated by the cardiomyocyte circadian clock and mediated by specific survival kinases. It is unknown whether such an innate time-dependent cardioprotection also affects larger species. Circadian variation in human cardioprotection during an AMI might have important clinical implications.

The aim of this study was to determine the impact of time-of-day of AMI onset on infarct size in patients with ST segment elevation myocardial infarction (STEMI).

\section{METHODS \\ Study population and variables analysed}

Consecutive patients with STEMI admitted to the Coronary Care Unit of Hospital Clínico San Carlos between March 2003 and September 2009 were retrospectively analysed. Demographic and clinical variables were prospectively recorded in 
our database and analysed post hoc as a proof-of-concept examination.

The time of AMI onset was carefully collected at admission to the hospital. Clinical characteristics such as Killip class, left ventricular ejection fraction (LVEF, evaluated by two-dimensional echocardiography), infarct location and cardiovascular outcomes were obtained during admission. Treatment of patients during admission was performed according to current clinical guidelines.

\section{Definitions}

STEMI was defined following current clinical practice guidelines: typical chest pain of $\geq 30 \mathrm{~min}$ and significant ST segment elevation $(\geq 0.1 \mathrm{mV}$ or $\geq 0.2 \mathrm{mV}$ on $\geq 2$ adjacent limb or precordial leads, respectively, or new left bundle-branch block) and confirmed by a rise in biomarkers. Infarct location was based on the culprit coronary artery. STEMI location was divided into anterior wall (left anterior descending coronary artery system occlusion) or any other location (left circumflex or right coronary artery systems). Time of onset of symptoms was obtained from the Coronary Care Unit medical history where this parameter is systematically recorded. The primary endpoint of the study was myocardial infarct size, which was defined according to peak creatine kinase (CK) and troponin I (TnI) levels. ${ }^{11}$ Cardiac enzyme levels were measured on admission and then every $4 \mathrm{~h}$ until a fall in both enzymes occurred. This methodology of peak enzyme determination is performed in all patients admitted to our Coronary Care Unit. CK determination was performed using the CK-Nac quantitative in vitro photometric reagent. The measurement of TnI was performed in all patients using the same commercial available kit (enzyme immunoanalysis).

Time-of-day of STEMI onset was first divided into four 6-hour periods according to previous studies ${ }^{2} 10{ }^{12-14}$ : (period 1: midnight-6:00 h; period 2: 6:00-noon (dark-to-light transition); period 3: noon-18:00 h; period 4: 18:00-midnight). The shape of the relationship between peak enzyme concentrations and time-of-day was analysed by multivariate regression splines. In an additional analysis, daytime was divided into two periods: dark-to-light transition period (6:00-noon) and other times of day (noon-6:00 h), in agreement with previous studies analysing the effect of circadian rhythm on the incidence of AMI. ${ }^{13} 14$ All results were studied in accordance with current data protection laws and following the ethical principles of the Declaration of Helsinki.

\section{Statistical analysis}

All the statistical analyses were performed using the open-source statistical scripting language $\mathrm{R}$ Version 2.11.0 (R Development Core Team).

The distributions of peak CK and TnI levels were positively skewed so a logarithmic transformation was used to make the data more symmetrical and homoscedastic. As a consequence, the CK and TnI concentrations were expressed in terms of geometric means (which are the anti-logarithm values of the mean of the logged data) and 95\% CI.

The trajectories of the peak enzyme concentrations across time have been characterised using multivariate regression splines. The spline covariates matrix used in the linear model was obtained using three cubic B-spline basis functions. The significance of the spline curves used in the study were determined by likelihood ratio tests (LRT), comparing nested multivariate linear models that only differed in the introduction of the spline time covariates. The order of the spline function (number of coefficients in each piecewise polynomial segment) and the position of the knots used to define the regression spline models corresponded to the best fitting model selected from a set of competing models fitted for different spline orders and vector of knots.

Peak CK and TnI concentration-adjusted differences comparing patients with STEMI onset at dark-to-light transition (6:00-noon) with the rest of the day were obtained using multivariate linear models. Owing to the log transformation of the outcome variables, the differences between peak enzyme concentrations were expressed in terms of geometric mean ratios.

All multivariate linear models were adjusted for potential confounder factors such as diabetes mellitus, previous treatment with $\beta$ blockers and ACE inhibitors, history of hypertension, AMI wall location, type of revascularisation, weekday/weekend period and year season of STEMI onset. Standard diagnostic checks on the residuals from the fitted models showed no evidence of any failure of the assumption of normality and homogeneity of the residual variance.

The remaining continuous variables used in the analysis were expressed as mean \pm SEM and categorical data as a percentage. The $\chi^{2}$ test was used for categorical comparisons of data. Differences in the means of continuous measurements were tested by either the Student $t$ test or ANOVA. A $p$ value of $<0.05$ (two-tailed) was considered significantly different.

\section{RESULTS}

A total of 950 consecutive patients with STEMI were admitted to the Coronary Care Unit between March 2003 and September 2009. In order to avoid potential bias, patients with a previous history of MI and those who were not revascularised by any means were systematically excluded from the analysis; 811 patients comprised the study population (causes for exclusion from the analysis in are shown in figure 1 in the online supplement).

Clinical variables from patients by the four 6-hour periods previously defined are shown in table 1 . The incidence of onset of AMI was significantly higher in the dark-to-light transition than in the other three groups (figure 1). Patients from the different time periods were balanced for all classical risk factors except hypertension which was significantly more frequent in patients from period 2 (6:00-noon). Anterior wall AMI was significantly more frequent in patients from periods 1 and 2 (midnight-noon), and time of ischaemia was significantly longer in period 1 (midnight-6:00 h) than in the other three time periods. Primary percutaneous coronary intervention (PCI) was consistently used more frequently as the form of revascularisation among all groups, although patients having an AMI in period 1 (midnight-6:00 h) were significantly more likely to undergo PCI than the other groups $(89 \%$ of patients in period 1 had PCI compared with $77 \%, 77 \%$ and $76 \%$ in the other three groups). There were no differences between groups in the rate of rescue PCI in patients revascularised by fibrinolysis. Time from reperfusion to peak rise in enzymes was similar in all four groups of patients (see figure 2 in online supplement).

LVEF was significantly lower in patients from periods 1 and 2 (midnight-noon) than in the other groups. Heart failure (Killip class $\geq 2$ during hospitalisation) was more frequent in period 2 (dark-to-light transition) than at any other time of the day (table 1).

To explore the relationship between peak enzyme concentrations and time, multivariate regression splines were used. 
Table 1 Baseline characteristics, management and outcomes of patients according to time-of-day infarction

\begin{tabular}{|c|c|c|c|c|c|c|}
\hline & Total & $\begin{array}{l}\text { Period 1: } \\
\text { Midnight-6:00 h }\end{array}$ & $\begin{array}{l}\text { Period 2: } \\
\text { 6:00-noon }\end{array}$ & $\begin{array}{l}\text { Period 3: } \\
\text { Noon-18:00 h }\end{array}$ & $\begin{array}{l}\text { Period 4: } \\
\text { 18:00-midnight }\end{array}$ & p Value \\
\hline Number of patients (\% of total) & $811(100 \%)$ & $141(17 \%)$ & $269(33 \%)$ & $240(29 \%)$ & $161(20 \%)$ & $<0.001$ \\
\hline \multicolumn{7}{|l|}{ Demographics } \\
\hline Male $(\%)$ & 78.4 & 84.4 & 78.4 & 74.2 & 79.5 & 0.130 \\
\hline Hypertension (\%) & 45.4 & 38.3 & 53.9 & 43.8 & 39.8 & 0.005 \\
\hline Dyslipidaemia (\%) & 41.1 & 40.4 & 42.0 & 42.1 & 38.5 & 0.883 \\
\hline Prior $\operatorname{Rx} \beta$ blockers (\%) & 5.4 & 4.1 & 6.9 & 5.3 & 4.2 & 0.591 \\
\hline Prior Rx statins (\%) & 13.9 & 12.5 & 16.5 & 16.1 & 7.9 & 0.419 \\
\hline Prior Rx ACE inhibitors (\%) & 19 & 11.4 & 27.1 & 18.9 & 12.7 & $<0.001$ \\
\hline Weekday (\%) & 74.1 & 75.9 & 76.6 & 71.3 & 72.7 & 0.516 \\
\hline Weekend (\%) & 25.9 & 24.1 & 23.4 & 28.8 & 27.3 & \\
\hline Spring (\%) & 27 & 24.1 & 26.4 & 29.6 & 26.7 & 0.482 \\
\hline Killip class $\geq 2(\%)$ & 29.4 & 28.8 & 34.6 & 24.3 & 29.1 & 0.089 \\
\hline Mean \pm SEM LVEF (\%) & $51.0 \pm 0.4$ & $49.9 \pm 0.9$ & $49.9 \pm 0.6$ & $52.3 \pm 0.6$ & $51.9 \pm 0.9$ & 0.025 \\
\hline Anterior wall location (\%) & 44.1 & 49.6 & 48.7 & 40.0 & 37.9 & 0.042 \\
\hline Multivessel disease (\%) & 12.9 & 14.9 & 12.3 & 10.8 & 15.5 & 0.478 \\
\hline \multicolumn{7}{|l|}{ Management } \\
\hline $\begin{array}{l}\text { Mean } \pm \text { SEM time from symptom } \\
\text { onset to reperfusion (min) }\end{array}$ & $229 \pm 6$ & $311 \pm 20$ & $226 \pm 10$ & $198 \pm 8$ & $215 \pm 12$ & $<0.001$ \\
\hline Primary PCI (\%) & 79.2 & 88.7 & 77.3 & 77.5 & 76.4 & 0.024 \\
\hline GP Illb/lla inhibitors & 46.1 & 50.4 & 43.5 & 44.2 & 49.7 & 0.398 \\
\hline \multicolumn{7}{|l|}{ In-hospital outcomes } \\
\hline Death $(\%)$ & 3.8 & 5.0 & 4.1 & 2.1 & 5.0 & 0.376 \\
\hline Reinfarction (\%) & 1.6 & 2.1 & 1.9 & 1.7 & 0.6 & 0.718 \\
\hline
\end{tabular}

ACE inhibitors, angiotensin converter enzyme inhibitors; CCU, coronary care unit; CK, creatine kinase; GM, geometric mean; GP, glycoprotein; LVEF, left ventricular ejection fraction; Ml, myocardial infarction; PCI, percutaneous coronary intervention; Rx, treatment; Tnl, troponin-l; VF, ventricular fibrillation; VT, ventricular tachycardia.

The spline curves included in the linear model were significant for both CK (LRT: $\left.\chi^{2}=10.4, \mathrm{df}=3, \mathrm{p}=0.015\right)$ and TnI (LRT: $\chi^{2}=10.9$, $\mathrm{df}=3, \mathrm{p}=0.012)$. The estimated curves for CK and TnI peaks showed parallel trajectories with a global maximum between 6:00 $\mathrm{h}$ and noon and a local minimum between noon and 18:00, as shown in figures 2 and 3 . For the variables used as potential confounders in the multivariate spline regression model, the localisation of the infarction (anterior wall location) showed a significant association with peak $\mathrm{CK}$ and TnI concentrations. A history of hypertension was inversely associated with CK peak concentration. Similarly, STEMI onset at weekends was associ-

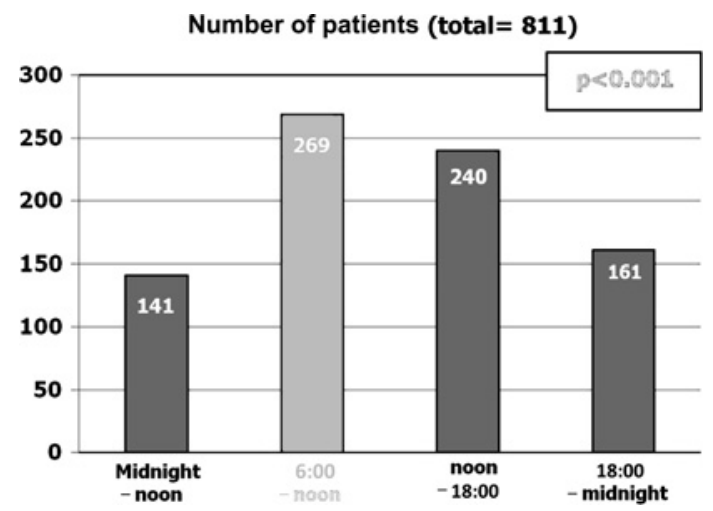

Figure 1 Distribution of patients (total number) according to time-ofday of AMI onset. ated with higher peak levels of CK than STEMI onset on weekdays. Neither a history of hypertension nor onset of STEMI at weekends was significantly associated with TnI levels. The F p values for partial sum of squares for all the confounders used in the multivariate analysis are shown in table 2 for both enzymes.

To further support the hypothesis that infarct size might be greater in the dark-to-light transition period (6:00-noon) as suggested by the estimated curves (figures 2 and 3), we compared the $\mathrm{CK}$ and TnI peak concentration-adjusted differences of patients with STEMI onset in the dark-to-light transition (6:00-noon) with those in the other time periods pooled together using multivariate regression analysis. This type of comparison (single period vs rest of the day) has been previously performed as a secondary analysis in different circadian evaluations by different authors. ${ }^{13} 14$ Table 3 summarises the demographic baseline characteristics for patients classified according to these two categories and shows that patients in the 6:00-noon period presented significantly more frequently with hypertension and diabetes mellitus. In addition, there was a trend $(p=0.066)$ towards more frequent anterior wall location of AMI in patients in the dark-to-light transition. The time of ischaemia and the revascularisation technique used (thrombolysis or PCI) were not statistically different in the two groups of patients.

LVEF was significantly lower in patients in the dark-to-light transition group than in the other group (49.9 \pm 1 vs $51.6 \pm 1$, $p=0.037$ ). Consequently, the rate of patients with heart failure was higher in patients with AMI onset in the 6:00-noon period (34.6\% vs $26.9 \%, p=0.024)$. 


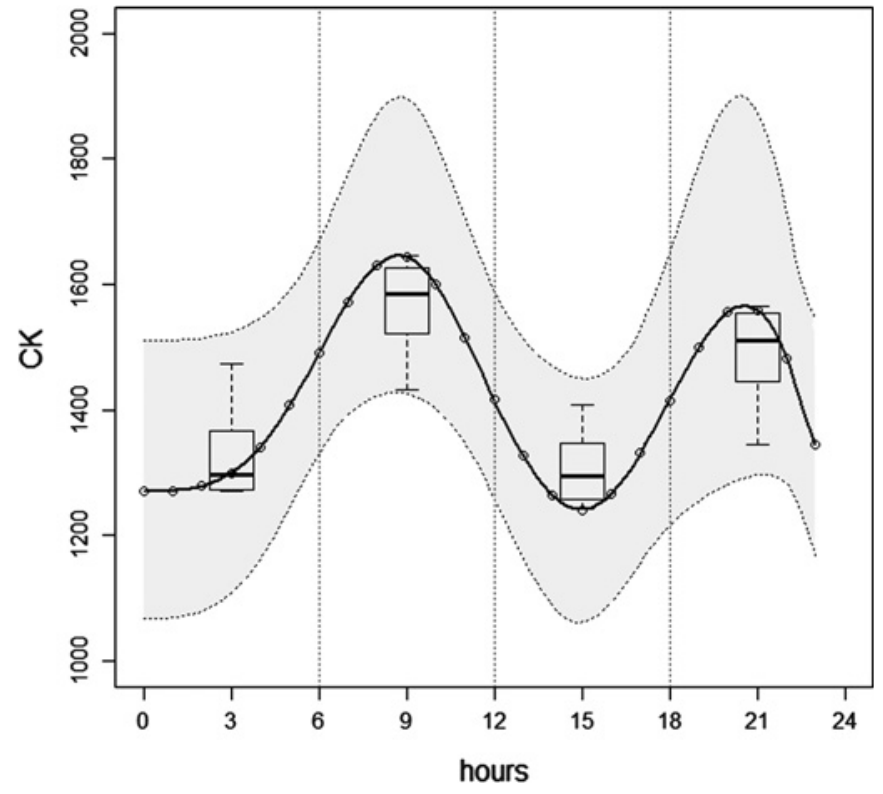

Figure 2 Adjusted geometric means and $95 \% \mathrm{Cl}$ of peak concentrations of creatine kinase (CK) estimated by regression splines. The fitted curve corresponds to an average ischaemia time of $30.48 \mathrm{~min}$ and reference levels for the rest of the confounders in the linear model (see text). The spline covariates matrix used in the linear model was obtained using three cubic B-spline basis functions defined for the vector of knots at times $t=(0,4,9,15,22,24,24)$. Boxplots represent curve estimated peak enzyme concentrations in each of the specified periods (midnight-6:00 h, 6:00-noon, noon-18:00 h, 18:00-midnight).

Infarct size was significantly larger in the dark-to-light transition group than in patients with STEMI onset at any other time of the day (CK: 1766.84 UI/1 (95\% CI 1554.34 to 2020.69)

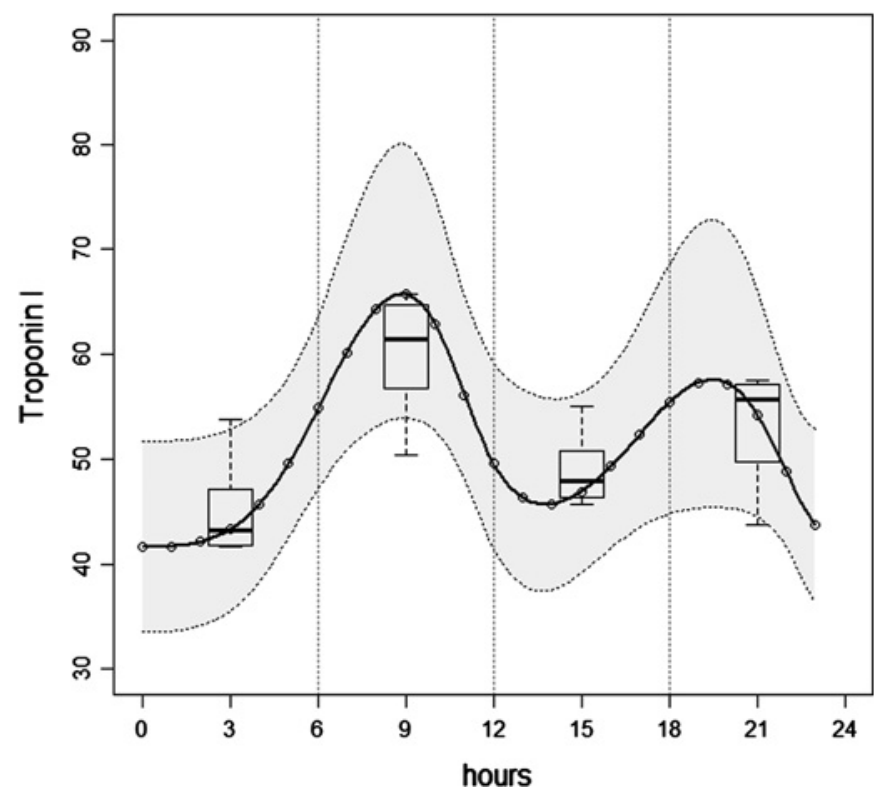

Figure 3 Adjusted geometric means and $95 \% \mathrm{Cl}$ of peak concentrations of troponin-I estimated by regression splines. The fitted curve corresponds to an average ischaemia time of $230.48 \mathrm{~min}$ and reference levels for the rest of the confounders in the linear model (see text). The spline covariates matrix used in the linear model was obtained using three cubic B-spline basis functions defined for the vector of knots at times $\mathrm{t}=(0,4,10,12,21,24,24)$. Boxplots represent curve estimated peak enzyme concentrations in each of the specified periods (midnight-6:00 h, 6:00-noon, noon-18:00 h, 18:00-midnight). vs 1517.21 UI/1 (95\% CI 1382.41 to 1649.81$)$; TnI: $66.65 \mathrm{ng} / \mathrm{ml}$ (95\% CI 56.47 to 78.76 ) vs $53.65 \mathrm{ng} / \mathrm{ml}$ (95\% CI 47.7 to 60.04 ).

The estimated adjusted differences obtained in the multivariate regression analysis showed that peak $\mathrm{CK}$ and $\mathrm{TnI}$ concentrations in patients with STEMI onset in the 6:00-noon period were $18.3 \%(p=0.031)$ and $24.6 \%(p=0.033)$ higher, respectively, than in those with STEMI onset at any other time of the day.

\section{DISCUSSION}

Cardiovascular physiology is significantly modulated by the circadian rhythm. It is well recognised that there are circadian oscillations in blood pressure, heart rate, cardiac output, myogenic tone, endothelial function and circulating levels of humoral factors. ${ }^{15}$ Data from epidemiological studies have shown that pathological states are also influenced by circadian fluctuations; previous reports have consistently found an increased incidence of AMI, sudden cardiac death, ventricular malignant arrhythmias, cardiogenic shock, stroke, acute aortic dissection and abdominal aortic aneurysm rupture during the early morning hours. ${ }^{216-19}$ In addition to the incidence of AMI, the effectiveness of thrombolytic therapy is also dependent on circadian time-of-day. ${ }^{20}$ Despite strong evidence for the time-ofday dependence of AMI incidence, which is higher in the sleepto-wake transition period, it is not known whether there are circadian oscillations in the human tolerance to I/R.

In this study we have retrospectively analysed the effect of the time-of-day onset of AMI on final infarct size in a singlecentre population of consecutive patients with first STEMI. The main findings of the study are: (1) there are significant circadian oscillations in infarct size in patients according to time-of-day of STEMI onset; and (2) onset of AMI in the dark-to-light transition period (6:00-noon) is associated with significantly larger MIs as evaluated by peak rise in enzyme concentrations. These associations were adjusted for clinical variables that could act as potential confounders. To the best of our knowledge, this is the first report of the impact of time-of-day of STEMI onset on infarct size. This could represent a variance in the tolerance to I/R (or spontaneous cardioprotection) according to time-of-day in humans with STEMI.

Numerous studies have evaluated the impact of time-of-day on the incidence of AMI. The interval limits chosen for circadian variation analyses have systematically been midnight, 6:00 h, noon and 18:00 h. ${ }^{2}{ }^{10}{ }^{12-14}$ In this study we first studied the demographic data of the population according to these established intervals and then analysed the relationship between peak enzyme concentrations and time by multivariate regression splines. The curves of peak enzyme concentrations further supported the choice of these time intervals (see figures 2 and 3 ).

Cohen et al performed a meta-analysis of more than 60000 patients and explored the time-of-day distribution of AMI onset. ${ }^{21}$ They found a consistently increased incidence of AMI in the dark-to-light transition period (6:00-noon), representing $32 \%$ of the entire meta-analysis population. Our data are in agreement with these results since $33 \%$ of the patients in our cohort had AMI onset in the early morning hours (6:00-noon). Interestingly, the distribution of our patients in the other time intervals (midnight-6:00 h: 17\%; noon-18:00 h: 29\%; 18:00midnight: $20 \%$, figure 1 ) matches that of previous studies. For instance, the time-of-day distribution of patients with AMI enrolled in the GISSI 2 study ( 11500 patients) analysed by Gnecchi-Ruscone and coworkers ${ }^{22}$ was almost identical to that of the present study (midnight-6:00 h: 17\%; 6:00-noon: $33 \%$; noon-18:00 h: 29\%; 18:00-midnight: $21 \%$ ). In agreement with previous studies, ${ }^{5}$ we found a higher incidence of hypertension 
Table 2 Partial sum of squares (type III) and F p values for the variables used in the multivariate spline regression model

\begin{tabular}{|c|c|c|c|c|c|c|c|c|}
\hline & \multicolumn{4}{|l|}{ CK } & \multicolumn{4}{|l|}{ Tnl } \\
\hline & Sum of squares type III & df & $\mathbf{F}$ & p Value & Sum of squares type III & df & $\mathbf{F}$ & p Value \\
\hline Diabetes mellitus & 1.06 & 1 & 1.245 & 0.265 & 1.05 & 1 & 0.737 & 0.398 \\
\hline Prior $\operatorname{Rx} \beta$ blockers & 0.28 & 1 & 0.327 & 0.568 & 1.66 & 1 & 1.163 & 0.288 \\
\hline Hypertension & 4.39 & 1 & 5.158 & 0.023 & 2.32 & 1 & 1.625 & 0.208 \\
\hline Time from symptom onset to reperfusion & 0.25 & 1 & 0.290 & 0.590 & 1.91 & 1 & 1.336 & 0.245 \\
\hline Anterior wall location & 7.43 & 1 & 8.719 & 0.003 & 18.39 & 1 & 12.872 & $<0.001$ \\
\hline Season & 4.49 & 3 & 1.758 & 0.154 & 7.57 & 3 & 1.766 & 0.158 \\
\hline Spline curve & 8.72 & 3 & 3.412 & 0.017 & 14.02 & 3 & 3.270 & 0.014 \\
\hline
\end{tabular}

Multivariate models adjusted for reference levels (no diabetes, no Rx with $\beta$-blocker, no Rx with ACE inhibitor, no history of hypertension, average time of ischaemia (230.48 min), anterior wall STEMI. Revascularisation by primary angioplasty, STEMI onset on weekend, STEMI onset in spring time.

ACE inhibitors, angiotensin converter enzyme inhibitors; AMI, acute myocardial infarction; df, degrees of freedom; F, F statistic; Rx, treatment; Tnl, troponin-I.

in the 6:00-noon period. Conversely, we found no differences between groups in the percentage of patients on $\beta$ blockers prior to the index event. Of note, it has been shown that treatment with $\beta$ blockers abolishes the dark-to-light transition peak incidence of both AMI and sudden death. ${ }^{14}{ }^{23}$ Even though the ultimate mechanism is unknown, it has been speculated that this protective effect of $\beta$-receptor antagonist therapy could be secondary to the blockade of the morning surge in sympathetic activity. In this regard, we have previously reported that $\beta$ blockers are strong cardioprotective agents. ${ }^{24}$

Table 3 Baseline characteristics, management and outcomes of patients according to time-of-day of infarction

\begin{tabular}{|c|c|c|c|}
\hline Demographics & 6:00-noon & Noon-6:00 h & p Value \\
\hline Mean \pm SEM age (years) & $62 \pm 0.8$ & $61 \pm 0.6$ & 0.542 \\
\hline Male (\%) & 78.4 & 78.4 & 0.993 \\
\hline Hypertension (\%) & 53.9 & 41.1 & 0.001 \\
\hline Dyslipidaemia (\%) & 42.0 & 40.6 & 0.699 \\
\hline Current smoking (\%) & 50.2 & 53.3 & 0.400 \\
\hline Diabetes mellitus (\%) & 25.3 & 17.2 & 0.006 \\
\hline Prior Rx $\beta$ blockers (\%) & 6.9 & 4.7 & 0.21 \\
\hline Prior Rx statins $(\%)$ & 16.5 & 12.6 & 0.371 \\
\hline Prior Rx ACE inhibitors (\%) & 27.1 & 15.1 & $<0.001$ \\
\hline Weekday (\%) & 76.6 & 72.9 & 0.269 \\
\hline Weekend (\%) & 23.4 & 27.1 & \\
\hline Spring (\%) & 26.4 & 27.3 & 0.944 \\
\hline Summer (\%) & 17.8 & 18.8 & \\
\hline Autumn (\%) & 27.5 & 27.5 & \\
\hline Winter (\%) & 28.3 & 26.4 & \\
\hline \multicolumn{4}{|l|}{ Clinical presentation } \\
\hline Killip class $\geq 2(\%)$ & 34.6 & 26.9 & 0.024 \\
\hline Mean \pm SEM LVEF (\%) & $49.9 \pm 0.6$ & $51.6 \pm 0.5$ & 0.037 \\
\hline Anterior wall location (\%) & 48.7 & 41.9 & 0.066 \\
\hline Multivessel disease (\%) & 12.3 & 13.3 & 0.685 \\
\hline \multicolumn{4}{|l|}{ Management } \\
\hline $\begin{array}{l}\text { Mean } \pm \text { SEM time from symptom } \\
\text { onset to reperfusion (min) }\end{array}$ & $226 \pm 20$ & $230 \pm 15$ & 0.725 \\
\hline Primary $\mathrm{PCI}(\%)$ & 77.3 & 80.1 & 0.364 \\
\hline GP Ilb/Illa inhibitors & 43.5 & 47.4 & 0.291 \\
\hline \multicolumn{4}{|l|}{ In-hospital outcomes } \\
\hline Death (\%) & 4.1 & 3.7 & 0.780 \\
\hline Reinfarction (\%) & 1.9 & 1.5 & 0.683 \\
\hline VF/VT (\%) & 10.4 & 7.2 & 0.118 \\
\hline
\end{tabular}

Patients were divided into two groups, those with myocardial infarction onset in the darkto-light transition (6:00-noon) and those with infarct onset at any other time of the day. ACE inhibitors, angiotensin converter enzyme inhibitors; CCU, coronary care unit;

CK, creatine kinase; GM, geometric mean; GP: glycoprotein; LVEF, left ventricular ejection fraction; $\mathrm{MI}$, myocardial infarction; $\mathrm{PCl}$, percutaneous coronary intervention; $\mathrm{Rx}$, treatment; Tnl, troponin-l; VF, ventricular fibrillation; VT, ventricular tachycardia.
The cardioprotective effect of $\beta$ blockers seems to be restricted to their administration prior to coronary reperfusion. ${ }^{25}$ In fact, treatment with $\beta$ blockers before an AMI has been shown to result in smaller infarcts ${ }^{26}$ and reduced myocardial injury during $\mathrm{PCI}^{27}$ These data suggest that these agents might reduce I/R injury and exert preconditioning-like properties. ${ }^{8} 28$ In this study, $\beta$ blockers and ACE inhibitors given before treatment showed a trend (albeit non-significant) for a negative correlation with infarct size. It is possible that a larger sample size would have resulted in a statistically significant difference. However, since the main objective of the study was to test whether timeof-day of STEMI onset has an impact on infarct size, all additional findings are speculative and should not be taken as final.

In addition to the higher incidence of hypertension in the dark-to-light transition period, we found a trend towards a higher incidence of diabetes mellitus in the same time period. The impact of diabetes on the morning peak incidence of AMI has been debated. Some authors have reported that the early morning peak incidence of AMI is attenuated in patients with diabetes ${ }^{29}$ while others have observed a similar peak incidence of AMI onset in the sleep-to-wake period. ${ }^{5}$ In addition to diabetes, the glucose levels at the time of AMI onset have been associated with poor outcomes in patients with diabetes. ${ }^{30}$ In our patients with diabetes the significantly higher incidence of AMI onset in the 6:00-noon period was not attenuated. The time from symptom onset to reperfusion was significantly different between the four periods with a longer time of ischaemia during the sleeping hours (midnight-6:00 h). This fact could be attributed to a delay in requesting medical assistance during the night and longer door-to-balloon time. ${ }^{31}$ However, MI size was clearly larger during the morning period even with a shorter mean time of ischaemia. Finally, we found significant differences in AMI location according to time-of-day. Patients with AMI onset in the sleeping and sleep-to-wake transition periods had a higher incidence of anterior wall AMI. These results are in agreement with previous studies, ${ }^{14}{ }^{32}$ but the mechanism responsible for the variation in distribution of AMI location during the daytime hours is not clear.

As expected, clinical outcomes, Killip class and LVEF agreed with the infarct size and AMI wall location. Heart failure and left ventricular dysfunction were more frequent in patients with STEMI onset in the period 6:00-noon. The fact that LVEF was reduced to a similar extent in the 6:00-noon and midnight-6:00 $\mathrm{h}$ groups despite a significant difference in MI size could be due to the similar incidence of anterior wall AMI and potentially larger viable stunned myocardium in the latter group. 
In addition to time-of-day of STEMI onset, we found that anterior wall STEMI location was significantly associated with greater infarct size, as evaluated by CK and TnI peak levels. The fact that both CK and TnI curves had the same pattern gives robustness to these results, supporting the finding that STEMI onset in the dark-to-light transition period and anterior wall AMI location are truly associated with larger infarcts.

The multivariate analysis showed two unexpected associations. Normotensive status prior to AMI (compared with hypertension) and STEMI onset at weekends were significantly associated with higher peak concentrations of CK. Strikingly, the peak TnI concentration was not significantly increased between the levels of these two variables. Because of the absence of parallelism between CK and TnI results in these variables, we cannot rule out the possibility that these might be false positive associations and therefore cannot be taken as a conclusive result. Further studies are needed to evaluate these results to provide a clear answer.

The mechanisms by which the circadian rhythm imposes an oscillating natural resistance to I/R (or, by contrast, reduced cardioprotection) are not fully understood. Given the important role in the final infarct size (after I/R) of both circulating and myocardial-resident factors, it is plausible to argue that factors influencing both states might play a role. The daily fluctuations in the systemic concentration of cortisol, circulating epinephrine levels, coronary flow, blood viscosity, endogenous thrombolytic activity and platelet aggregability are well known. ${ }^{3} 3334$ The fact that the early morning clustering of onset of acute cardiovascular events concurs with the morning circadian increase in platelet aggregability, heart rate, blood pressure and other factors supports the role of systemic factors in the increased incidence of AMI in the sleep-to-wake transition period. In agreement with these data, we also found an increased incidence of AMI between 6:00 $\mathrm{h}$ and noon.

The first examination on the effect of time-of-day on infarct size was recently undertaken by Durgan et al. ${ }^{10}$ They observed significant fluctuations in infarct size depending on the time-ofday at which I/R was performed in rodents; larger infarcts were seen when the coronary insult occurred at the normalised sleepto-wake transition. ${ }^{10}$ In the present study we observed a similar pattern of infarct size according to the time of AMI onset (figures 2 and 3), suggesting that the fluctuating cardioprotection seen in rodents is also present in humans. In contrast with the preclinical findings of Durgan et $a l^{10}$ we found a consistent second peak in infarct size in the 18:00-midnight period (figures 2 and 3). This second peak was observed when infarct size was evaluated either by CK or TnI peak concentrations, providing robustness to the finding. The appearance of a second (albeit smaller) increase in infarct size suggests that the oscillations in infarct size observed are dictated to some extent by the circadian clock. ${ }^{4}$ Interestingly, De Luca et $a l^{32}$ studied the effect of time of reperfusion by primary PCI (rather than time of AMI onset) on MI size and observed that patients treated between 4:00 $\mathrm{h}$ and 8:00 $\mathrm{h}$ had a trend towards larger infarctions. Unfortunately, MI size was not normalised to important clinical variables such as time of ischaemia (which is significantly larger in this time window). In contrast to this study, the present proof-of-principle analysis was designed to determine whether time of symptom onset rather than time of treatment plays a role in the spontaneous tolerance to I/R (cardioprotection), and the time intervals were chosen according to numerous previous clinical papers analysing circadian oscillations and cardiovascular disease. ${ }^{2} 1012-14$
Final myocardial infarct size has been shown to be a strong predictor of cardiovascular events. ${ }^{35}$ As a consequence, infarct size is increasingly used as a primary end point in clinical trials. ${ }^{36}$ This study shows a time-dependent tolerance to I/R in humans. The correlation of MI size and time-of-day was independent of other important variables. If confirmed in prospective studies, these results might have significant clinical implications in the analysis of clinical trials of cardioprotection. In such studies, the variable 'time of AMI onset' has not been taken into account and might have represented a significant bias in the results arising from those trials.

\section{Limitations}

Even though the data collection was done prospectively, the study was performed post hoc as a hypothesis-generating study. The retrospective nature represents the main limitation of the present work. Myocardial infarct size was assessed by a surrogate marker-namely, peak rise in enzyme levels. Even though it has been systematically reported that this methodology is accurate and correlates with other methods, a direct evaluation of MI size (ie, magnetic resonance imaging or myocardial scintigraphy) would have evaluated MI size more precisely. Finally, given that the incidence of arrhythmias and sudden death is known to be higher in the early morning hours, survivor bias in the group of patients with STEMI onset in the dark-to-light transition period cannot be completely ruled out.

\section{CONCLUSIONS}

In this retrospective study we evaluated the impact of time-ofday of AMI onset on infarct size. We found an independent circadian oscillating pattern in infarct size across the time-ofday. Patients with STEMI onset in the dark-to-light transition period (6:00-noon) had significantly larger myocardial infarctions than those with symptom onset at any other time of the day. Overall, there is an expected increase of about $20 \%$ in infarct size in patients with STEMI onset in the dark-to-light transition period compared with any other time of day.

The correlation between time of AMI onset and MI size was independent of important clinical variables, reinforcing the critical impact of time-of-day on human tolerance to I/R. In addition, and confirming previous studies, we found a significantly higher incidence of AMI and anterior wall AMI in the early morning hours. Anterior wall AMI was also found to be significantly associated with larger infarcts.

This study is the first to document circadian fluctuations in spontaneous cardioprotection in humans with STEMI. These results advocate the inclusion of 'time of AMI onset' as an important variable in clinical trials testing novel cardioprotective strategies with MI size as an end point.

Acknowledgements The authors thank Drs Valentín Fuster and Ginés Sanz, CNIC, Madrid for their critical review of the findings and the manuscript.

\section{Competing interests None.}

Ethics approval This study was conducted with the approval of the Hospital Clínico San Carlos.

Provenance and peer review Not commissioned, externally peer reviewed.

\section{REFERENCES}

1. Rudic RD. Time is of the essence: vascular implications of the circadian clock. Circulation 2009;120:1714-21.

2. Muller JE, Tofler GH, Stone PH. Circadian variation and triggers of onset of acute cardiovascular disease. Circulation 1989;79:733-43. 
3. Ohkura N, Oishi $K$, Sudo $T$, et al. CLOCK regulates circadian platelet activity. Thromb Res 2009; 123:523-7.

4. Durgan DJ, Young ME. The cardiomyocyte circadian clock: emerging roles in health and disease. Circ Res 2010;106:647-58.

5. Hjalmarson A, Gilpin EA, Nicod P, et al. Differing circadian patterns of symptom onset in subgroups of patients with acute myocardial infarction. Circulation 1989:80:267-75.

6. Ridker PM, Manson JE, Buring JE, et al. Circadian variation of acute myocardial infarction and the effect of low-dose aspirin in a randomized trial of physicians. Circulation 1990;82:897-902.

7. Hausenloy DJ, Yellon DM. New directions for protecting the heart against ischaemia-reperfusion injury: targeting the Reperfusion Injury Salvage Kinase (RISK)-pathway. Cardiovasc Res 2004;61:448-60.

8. Ibanez B, Fuster V, Jimenez-Borreguero J, et al. Lethal myocardial reperfusion injury: a necessary evil? Int J Cardio/ Published Online First: 18 November 2010. doi:10.1016/j.ijcard.2010.10.056. PMID: 21093938.

9. litaka C, Miyazaki K, Akaike T, et al. A role for glycogen synthase kinase-3beta in the mammalian circadian clock. J Biol Chem 2005;280:29397-402

10. Durgan DJ, Pulinilkunnil T, Villegas-Montoya C, et al. Short communication: ischemia/reperfusion tolerance is time-of-day-dependent: mediation by the cardiomyocyte circadian clock. Circ Res 2010;106:546-50.

11. Chia S, Senatore F, Raffel OC, et al. Utility of cardiac biomarkers in predicting infarct size, left ventricular function, and clinical outcome after primary percutaneous coronary intervention for ST-segment elevation myocardial infarction. JACC Cardiovasc Interv 2008;1:415-23.

12. Muller JE, Ludmer PL, Willich SN, et al. Circadian variation in the frequency of sudden cardiac death. Circulation 1987;75:131-8.

13. Muller JE, Stone PH, Turi ZG, et al. Circadian variation in the frequency of onset of acute myocardial infarction. N Engl J Med 1985;313:1315-22.

14. Willich SN, Linderer T, Wegscheider $K$, et al. Increased morning incidence of myocardial infarction in the ISAM Study: absence with prior beta-adrenergic blockade. ISAM Study Group. Circulation 1989;80:853-8.

15. Richards AM, Nicholls MG, Espiner EA, et al. Diurnal patterns of blood pressure, heart rate and vasoactive hormones in normal man. Clin Exp Hypertens 1986;8:153-66.

16. Anon. Morning peak in the incidence of myocardial infarction: experience in the ISIS-2 trial. ISIS-2 (Second International Study of Infarct Survival) Collaborative Group. Eur Heart J 1992;13:594-8.

17. Elliott WJ. Circadian variation in the timing of stroke onset: a meta-analysis. Stroke 1998;29:992-6.

18. Englund A, Behrens S, Wegscheider $K$, et al. Circadian variation of malignant ventricular arrhythmias in patients with ischemic and nonischemic heart disease after cardioverter defibrillator implantation. European 7219 Jewel Investigators. J Am Coll Cardiol 1999;34:1560-8.

19. Manfredini R, Boari B, Gallerani M, et al. Chronobiology of rupture and dissection of aortic aneurysms. J Vasc Surg 2004;40:382-8.

20. Kono T, Morita $H$, Nishina $T$, et al. Circadian variations of onset of acute myocardial infarction and efficacy of thrombolytic therapy. J Am Coll Cardiol 1996;27:774-8.
21. Cohen MC, Rohtla KM, Lavery CE, et al. Meta-analysis of the morning excess of acute myocardial infarction and sudden cardiac death. Am J Cardiol 1997;79:1512-16.

22. Gnecchi-Ruscone T, Piccaluga E, Guzzetti S, et al. Morning and Monday: critical periods for the onset of acute myocardial infarction. The GISSI 2 Study experience. Eur Heart J 1994:15:882-7.

23. Peters RW, Muller JE, Goldstein S, et al. Propranolol and the morning increase in the frequency of sudden cardiac death (BHAT Study). Am J Cardiol 1989:63:1518-20.

24. Ibanez B, Prat-Gonzalez S, Speidl WS, et al. Early metoprolol administration before coronary reperfusion results in increased myocardial salvage: analysis of ischemic myocardium at risk using cardiac magnetic resonance. Circulation 2007; 115:2909-16

25. Ibanez B, Cimmino G, Prat-Gonzalez S, et al. The cardioprotection granted by metoprolol is restricted to its administration prior to coronary reperfusion. Int J Cardiol. 2011;147:428-32.

26. Halkin A, Grines CL, Cox DA, et al. Impact of intravenous beta-blockade before primary angioplasty on survival in patients undergoing mechanical reperfusion therapy for acute myocardial infarction. J Am Coll Cardiol 2004;43:1780-7.

27. Sharma SK, Kini A, Marmur JD, et al. Cardioprotective effect of prior beta-blocker therapy in reducing creatine kinase-MB elevation after coronary intervention: benefit is extended to improvement in intermediate-term survival. Circulation 2000:102:166-72.

28. Ibanez B, Cimmino G, Badimon JJ. Myocardial reperfusion injury. N Eng/ J Med 2007:357:2409-10.

29. Rana JS, Mukamal KJ, Morgan JP, et al. Circadian variation in the onset of myocardial infarction: effect of duration of diabetes. Diabetes 2003;52:1464-8.

30. Vivas D, Garcia-Rubira JC, Gonzalez-Ferrer JJ, et al. [Prognostic value of first fasting glucose measurement compared with admission glucose level in patients with acute coronary syndrome]. Rev Esp Cardiol 2008;61:458-64.

31. Zahn R, Schiele R, Seidl K, et al. Daytime and nighttime differences in patterns of performance of primary angioplasty in the treatment of patients with acute myocardial infarction. Maximal Individual Therapy in Acute Myocardial Infarction (MITRA) Study Group. Am Heart J 1999:138:1111-17.

32. De Luca G, Suryapranata H, Ottervanger JP, et al. Circadian variation in myocardia perfusion and mortality in patients with ST-segment elevation myocardial infarction treated by primary angioplasty. Am Heart J 2005;150:1185-9.

33. Linsell CR, Lightman SL, Mullen PE, et al. Circadian rhythms of epinephrine and norepinephrine in man. J Clin Endocrinol Metab 1985:60:1210-15.

34. Tofler GH, Brezinski D, Schafer Al, et al. Concurrent morning increase in platelet aggregability and the risk of myocardial infarction and sudden cardiac death. $N$ Engl J Med 1987;316:1514-18.

35. Kelle S, Roes SD, Klein C, et al. Prognostic value of myocardial infarct size and contractile reserve using magnetic resonance imaging. J Am Coll Cardiol 2009:54:1770-7.

36. Mewton N, Croisille P, Gahide G, et al. Effect of cyclosporine on left ventricular remodeling after reperfused myocardial infarction. J Am Coll Cardiol 2010:55:1200-5. 